\title{
Note the descending aorta: predictors of postoperative major adverse aortic event in pure acute type $A$ intramural hematoma
}

\author{
Myeong Su Kim ${ }^{1}$, Tae-Hoon Kim ${ }^{1}$, Ha Lee ${ }^{1}$, Suk-Won Song ${ }^{1}$, Woon Heo ${ }^{2}$, Seo-A Sim ${ }^{1}$, Kyung-Jong Yoo ${ }^{3}$ \\ ${ }^{1}$ Department of Cardiovascular Surgery, Gangnam Severance Hospital, Yonsei University College of Medicine, Seoul, Republic of Korea; \\ ${ }^{2}$ Department of Thoracic and Cardiovascular Surgery, Haeundae Paik Hospital, Inje University College of Medicine, Busan, Republic of Korea; \\ ${ }^{3}$ Department of Cardiovascular Surgery, Yonsei Cardiovascular Hospital, Severance Hospital, Yonsei University College of Medicine, Seoul, Republic \\ of Korea \\ Contributions: (I) Conception and design: All authors; (II) Administrative support: TH Kim, W Heo, KJ Yoo; (III) Provision of study materials or \\ patients: All authors; (IV) Collection and assembly of data: MS Kim, TH Kim, H Lee, SW Song, SA Sim; (V) Data analysis and interpretation: All \\ authors; (VI) Manuscript writing: All authors; (VII) Final approval of manuscript: All authors. \\ Correspondence to: Suk-Won Song, MD, PhD. Department of Cardiovascular Surgery, Gangnam Severance Hospital, Yonsei University College of \\ Medicine, 211 Enoju-ro Gangnam-gu, Seoul 06273, Republic of Korea. Email: sevraphd@yuhs.ac.
}

Background: Intramural hematomas (IMHs) may originate from small intimal tears. Although most surgeries for acute type A IMH are conventionally performed solely at the proximal aorta, regardless of the primary intimal tear site, the remnant aortic remodeling stays important during the follow-up period after surgery.

Methods: Forty-seven patients with "pure" acute type A IMHs who underwent surgery from January 2008 to December 2019 were retrospectively analyzed. Acute type A IMH in the entire region without penetrating aortic ulcer (PAU) and aortic dissection (AD), which upon initial computed tomography (CT), can be considered as an intimal tear site, was defined as "pure" type. The maximal diameter of the aorta, maximal thickness of the IMH, and hematoma thickness ratio (HTR) of the ascending and descending aortae were measured from the preoperative computed tomographic scan. The hematoma thickness index was defined as the HTR of the descending aorta divided by that of the ascending aorta. Major adverse aortic events (MAAEs) were defined as AD, rupture, or newly developed PAU and aortic death. Predictors for postoperative MAAEs were analyzed using preoperative computed tomographic findings.

Results: The measurements of the descending aorta were larger and those of the ascending aorta were smaller in the MAAEs group, than in the corresponding other. The hematoma thickness index was significantly higher in the group with MAAEs, than in the group without; this variable was an independent predictor of MAAEs. During surgery, intimal tears were found in 16/47 (34\%) patients. The hematoma thickness index was significantly smaller in the group with intimal tears than in the group without the tears. The aortic measurement appears to reflect the tear site.

Conclusions: Hematoma thickness index was an independent predictor of MAAE after acute type A IMH surgery. Long-term periodical follow-up with early reintervention may, therefore, be necessary to improve outcome in these patients. As the optimal treatment method is still controversial, inferring the location of the primary tear through the hematoma thickness index can be helpful in determining the treatment method.

Keywords: Acute type A intramural hematoma (ATAIMH); major adverse aortic event (MAAE); hematoma thickness ratio (HTR); surgery; computed tomography (CT)

Submitted Apr 17, 2021. Accepted for publication Jul 14, 2021.

doi: $10.21037 /$ jtd-21-674

View this article at: https://dx.doi.org/10.21037/jtd-21-674

(C) Journal of Thoracic Disease. All rights reserved. 


\section{Introduction}

Aortic intramural hematoma (IMH) is a condition that covers the acute aortic syndrome spectrum, characterized by the absence of intimal tear, and thereby, the continuous communication between the true and false lumens. IMH was first described by Krukenberg in 1920 as an "aortic dissection (AD) without an intimal tear" (1). Early studies did not detect an intimal tear or flow communication in IMH patients (2); hence, the hemorrhage could be the result of a ruptured vasa vasorum. However, few recent studies discussed intimal tears that were identified with more sensitive imaging modalities in numerous IMH cases $(3,4)$. Moreover, Uchida et al. (5) and Park et al. (6) noted that intimal tears were found in many IMH patients intraoperatively. This suggests that IMH may result from small intimal tears rather than a rupture of vasa vasorum. Therefore, the American College of Cardiology (ACC) Foundation and the American Heart Association (AHA) guidelines state that "When the term IMH is used strictly, no intimal defect such as a tear or an ulcer is present. But in practice, the term is used loosely to mean a thrombosed false lumen regardless of a small defect" (7). The established treatment for acute type A AD involves tear-oriented surgery, resection of the primary intimal tear, and graft replacement (8). Similarly, the treatment goal for acute type $\mathrm{B} \mathrm{AD}$ is the coverage of the primary intimal tear through thoracic endovascular aortic repair (TEVAR) (9). However, despite its similarities to $\mathrm{AD}$, its management is controversial, especially for type A IMH (10).

Although locating primary intimal tears of an acute type A intramural hematoma (ATAIMH) in preoperative computed tomography (CT) scans is difficult, most surgeries are performed on the proximal aorta (ascending aorta and arch). Grimm et al. (11) reported the novel insights into the mechanism and treatment of IMH. In particular setting that a primary tear is not detectable during open surgery for IMH, it remains unclear whether the predominant mechanism of this life-threatening disease has been adequately addressed. Therefore, these patients are at risk of having additional adverse events associated with an unidentified lesion in other aortic segments distal to the treated area. ATAIMH in the entire region without penetrating aortic ulcer (PAU) and $\mathrm{AD}$ (including focal lesions), which upon initial CT, can be considered as an intimal tear site, was defined as "pure" ATAIMH. Our study aimed to report early and late outcomes after surgery for "pure" ATAIMH. Further, we evaluated the prognostic factors of major adverse aortic events (MAAEs) following surgery for "pure" ATAIMH, as analyzed from the preoperative CT scan findings.

We present the following article in accordance with the STROBE reporting checklist (available at https://dx.doi. org/10.21037/jtd-21-674).

\section{Methods}

\section{Ethical statement}

The study was conducted in accordance with the Declaration of Helsinki (as revised in 2013). The study was approved by the Institutional Review Board of the Gangnam Severance Hospital, Yonsei University College of Medicine (Yonsei Institutional Review Board approval no. 3-2020-0317), and the requirement for obtaining individual consent was waived in this retrospective analysis.

\section{Study design}

This study is a retrospective analysis of 407 patients with ATAIMH who were admitted to our hospital between January 2008 and December 2019. Patients with Marfan syndrome, malignancy, prominent PAU, and AD on CT were excluded; consequently, only those with "pure" ATAIMH were included. All patients underwent surgery immediately after the diagnosis.

\section{Definitions of IMH and PAU}

The presence of a hematoma within the aortic wallrecognizable as a crescent or circular local aortic wall thickening - and absence of a visible intimal flap or tear were the prerequisites for diagnosing IMH. A PAU was defined as a deep ulcerated lesion in the thickest part of IMH within the involved aorta (12).

\section{Measurement of aortic diameter}

On preoperative CT, the maximal diameter of the aorta, maximal thickness of the IMH (HT), and hematoma thickness ratio [HTR (hematoma thickness/aortic diameter)] were evaluated in the ascending and descending aortae (Figure 1). In the descending aorta, additional measurements were obtained at the level of inferior vena cava (IVC) and celiac axis (CA), in same manner. HTR of the descending aorta (dHTR)/HTR of the ascending aorta 
A

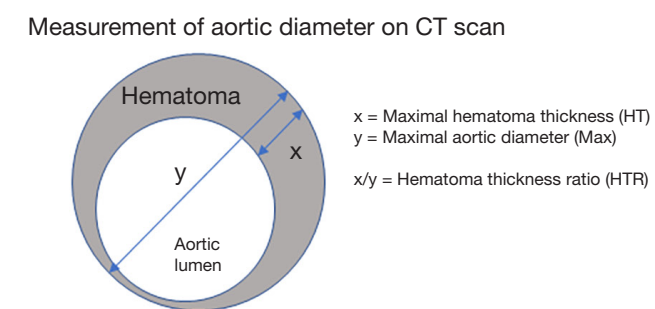

Cross section of aorta involved intramural hematoma

${ }^{*} \mathrm{dHTR} /{ }^{*} \mathrm{aHTR}=\mathrm{HT}$ index_Max

*dHTR_IVC/*aHTR $=$ HT index_IVC

*dHTR_CA/*aHTR $=$ HT index_CA

${ }^{*} \mathrm{a}=$ ascending aorta; $\mathrm{d}=$ descending aorta
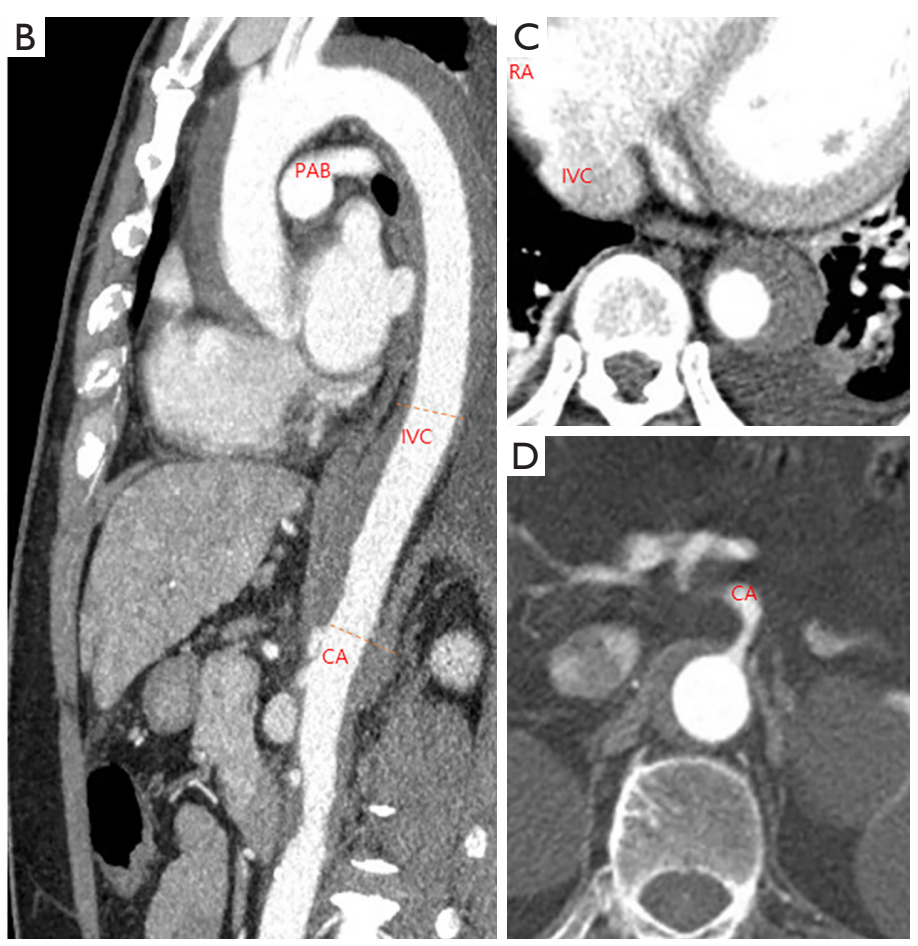

Figure 1 Measurement of diameters of the aorta and hematoma thickness using CT scans. (A) Schematic figure of the key concept of this study; (B-D) in the descending aorta, maximal diameter and hematoma thickness were mostly measured at the level of the PAB. Additional measurements were taken at the level of the IVC-RA junction and CA. CT, computed tomography; PAB, pulmonary artery bifurcation; CA, celiac axis; RA, right atrium; IVC, inferior vena cava.

(aHTR) of each level was named the HT index.

\section{Endpoint of study}

Forty-seven "pure" ATAIMH patients were divided according to the presence or absence of MAAEs, and preoperative CT findings were compared. The primary clinical endpoint was freedom from MAAEs. MAAEs were defined as $\mathrm{AD}, \mathrm{PAU}$, aortic rupture, aortic reintervention and aortic death. Indications of re-intervention included symptomatic MAAEs, asymptomatic newly developed PAU $>2 \mathrm{~cm}$ width and $>1 \mathrm{~cm}$ depth, and rapid growing aortopathy. Secondarily, we also investigated the predictors of MAAEs based on the findings on preoperative CT. The time-to-MAAE was defined as the time between the surgery and either MAAEs or death without MAAEs or censoring (alive without MAAEs at the last time point).

\section{Surgical technique}

During the surgery, moderate hypothermic circulatory arrest was induced for the distal aortic anastomosis in patients undergoing partial and total arch replacement. The right axillary artery was generally cannulated, while some cases needed femoral artery cannulation. After the rectal temperature of patients was sufficiently lowered for circulatory arrest, the adventitia of the ascending aorta was longitudinally incised, while the cardiopulmonary bypass flow was lowered to $1 \mathrm{~L} / \mathrm{min}$. The hematoma in the false lumen was gently removed with suction. Fresh blood gushing out from the false lumen was considered a strong sign of an intimal defect in a nearby segment of the aorta. After this maneuver, the true lumen of the ascending aorta was incised under total circulatory arrest and the inner wall was thoroughly inspected to find an intimal defect. The remaining thoracic aorta was also inspected. Before the proximal and distal anastomosis, neo-media formation was performed with Teflon felt.

\section{Statistical analyses}

Pearson's Chi-squared test and Fisher's exact test were 


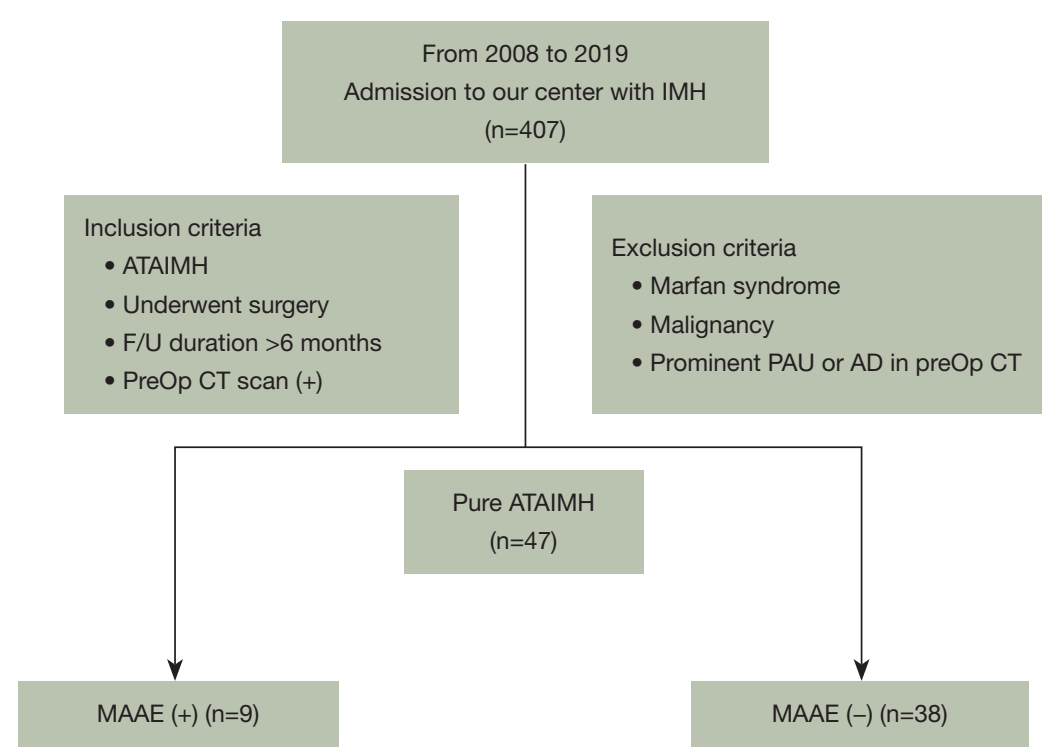

Figure 2 Flow-diagram of this study. AD, aortic dissection; ATAIMH, acute type A intramural hematoma; CT, computed tomography; F/U, follow-up; IMH, intramural hematoma; MAAE, major adverse aortic event; PAU, penetrating aortic ulcer; PreOp, preoperative.

used to compare the frequencies between patients with and without MAAEs. Continuous variables were examined for normality using the Kolmogorov-Smirnov test. These data are expressed as means (standard deviations), and compared using the Student's $t$-test. Non-normal continuous variables are expressed as medians [25th, 75 th interquartile points] and compared using the Mann-Whitney $\mathrm{U}$ test. Categorical variables are expressed as numbers (percentages). The time-related event was an MAAE, and freedom from this event was estimated using the Kaplan-Meier method and compared using the log-rank test. Maximally-selected rank statistics analysis using R package [Torsten Hothorn (2017) maxstat: Maximally Selected Rank Statistics. R package version $0.7-25$ ] was performed to determine the best cutoff value of each aortic measurement in predicting MAAEs. The rates of freedom from MAAEs and overall survival of all patients are presented. The independent risk factors of MAAEs were determined using the Cox proportional hazards regression analysis. For this analysis, variables with $\mathrm{P}<0.15$ in the univariate analysis were included. The results of the analysis are presented as hazard ratios (HRs) with corresponding $95 \%$ confidence intervals (CIs). The statistical analyses were performed using the statistical software R v4.0.4 (R Foundation for Statistical Computing, Vienna, Austria) and SPSS v23.0.0.0 for Windows (IBM Corp., Armonk, NY, USA).

\section{Results}

\section{Baseline characteristics}

Among the 407 patients admitted to our hospital with ATAIMH, 47 patients remained according to exclusion and inclusion criteria. They were divided according to the presence or absence of MAAEs, and the preoperative CT findings were compared (Figure 2). The baseline characteristics of patients and surgical outcomes are summarized in Table 1. The MAAE group comprised nine patients, of whom five were male, and the mean age was 65.44 (12.35) years. Hypertension was the most common comorbidity in both groups. There were no statistically significant differences in age, sex, or past medical history between the two groups.

\section{Preoperative CT findings}

As shown in Table 1, preoperative CT of the MAAE group demonstrated trends of lower maximal diameter of the ascending aorta (aMax), hematoma thickness of the ascending aorta (aHT), aHTR and trends of higher maximal diameter of the descending aorta (dMax), thickness of the descending aorta (dHT), and dHTR. There were no statistically significant differences between the groups. HT index_Max (dHTR/aHTR) was significantly higher 
Table 1 Baseline characteristics and surgical outcomes

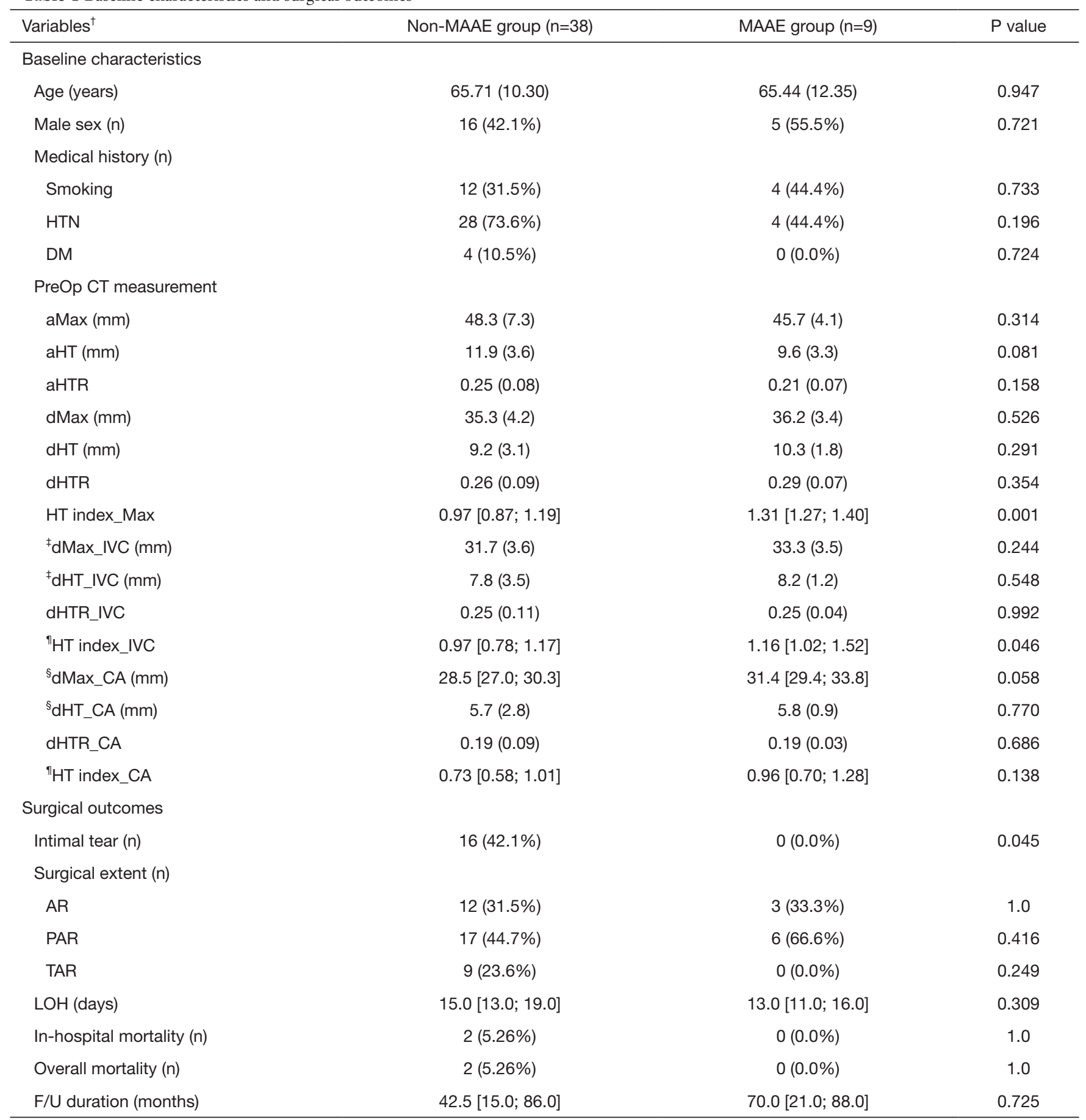

${ }^{\dagger}$, values expressed as means (standard deviations) for normally distributed continuous data, medians [IQRs: 25th percentile; 75th percentile] for non-normal distributed continuous data, or numbers (percentages) for categorical data; ${ }^{\ddagger}$, measurements were taken at the level of junction of inferior vena cava and right atrium; ${ }^{\S}$, measurements were taken at the level of celiac axis; ", HT index_IVC = dHTR_ IVC/aHTR; HT index_CA = dHTR_CA/aHTR. aHT, hematoma thickness of ascending aorta; aHTR, hematoma thickness ratio of ascending aorta; aMax, maximal diameter of ascending aorta; AR, ascending aorta replacement; CA, celiac axis; CT, computed tomography; dHT, hematoma thickness of descending aorta; dHTR, hematoma thickness ratio of descending aorta; DM, diabetes mellitus; dMax, maximal diameter of descending aorta; F/U, follow-up; HTN, hypertension; IVC, inferior vena cava; LOH, length of hospitalization; MAAE, major adverse aortic event; Op, operation; PAR, partial arch replacement; TAR, total arch replacement. 

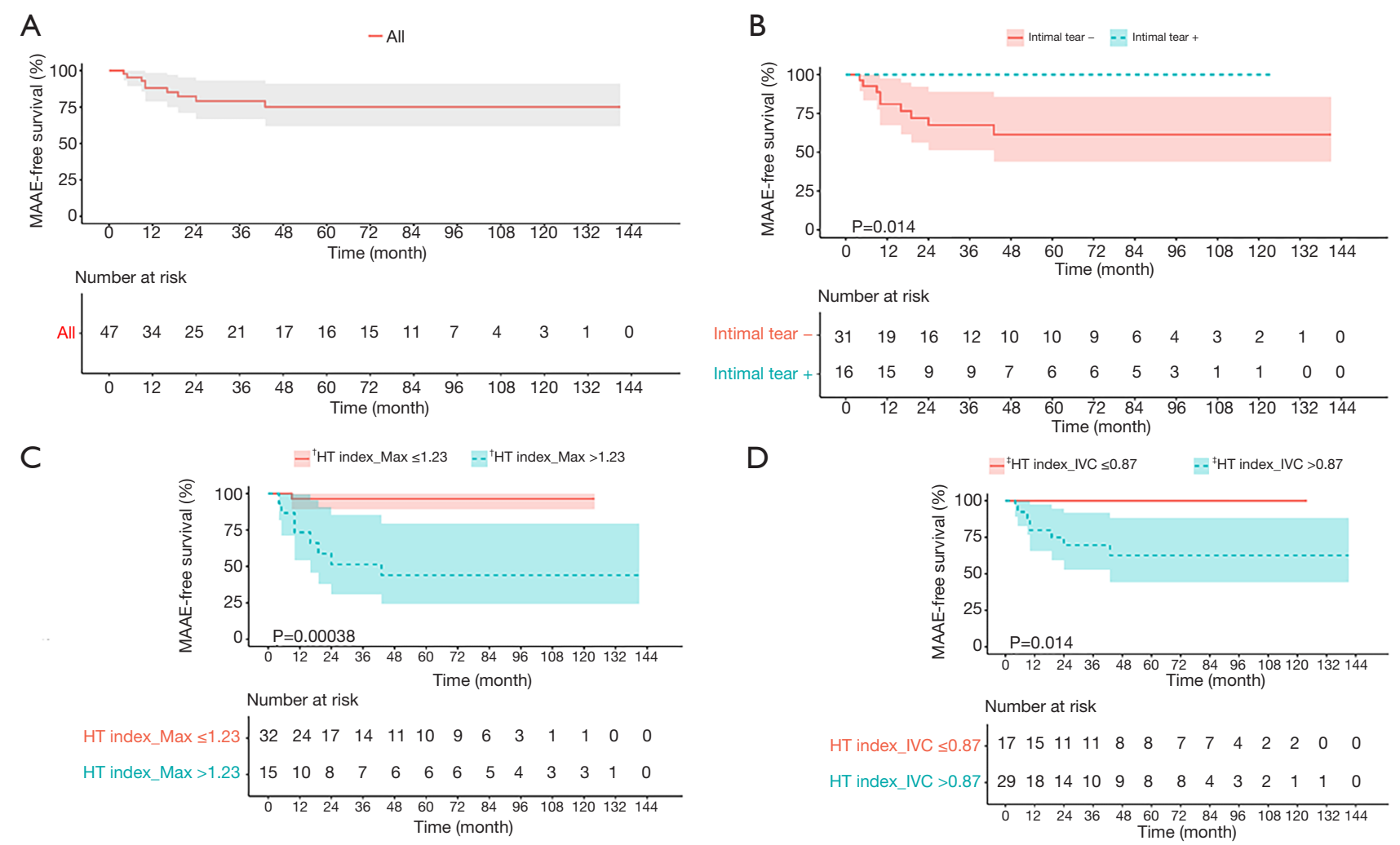

Figure 3 Kaplan-Meier curves of freedom from MAAEs. (A) MAAE-free survival rate of total patients; (B) MAAE-free survival rate according to intimal tear; (C) MAAE-free survival rate according to HT index_Max; (D) MAAE-free survival rate according to HT index_ IVC. Freedom from MAAE was estimated using the Kaplan-Meier method and compared using the log-rank test. Using maximally selected rank statistics analysis with $\mathrm{R}$ package to evaluate best cut off value for predicting MAAE. ${ }^{\dagger}, \mathrm{HT}$ index_Max $=\mathrm{dHTR} / \mathrm{aHTR}{ }^{\ddagger}, \mathrm{HT}$ index $\_$ IVC = dHTR_IVC/aHTR. HT, maximal hematoma thickness; IVC, inferior vena cava; MAAE, major adverse aortic event.

in the MAAE group than in the non-MAAE group $\{1.31$ [1.27; 1.40] vs. 0.97 [0.87; 1.19], respectively, $\mathrm{P}=0.001\{$. In most of the patients, maximal diameter was at the level of pulmonary artery bifurcation. As for the level of the IVC and CA, the measurements tended to be higher in MAAE group, when compared with the measured values in the ascending aorta. The HT index_IVC showed a significant difference \{non-MAAE $v s$. MAAE; 0.97 [0.78; 1.17] vs. 1.16 $[1.02 ; 1.52], \mathrm{P}=0.046\}$.

\section{Operative outcomes}

Intimal tears were noted during surgery in 16 (34\% of total patients) patients, all of whom were in the non-MAAE group (non-MAAE $v s$. MAAE group; $\mathrm{n}=16 ; 43.2 \%$ vs. $\mathrm{n}=0$; $0 \%$, respectively; $\mathrm{P}=0.01$ ). Total arch replacement was performed in 9 patients, and all were in the non-MAAE group. The average length of hospitalization was 15.0 [13.0; 19.0] days vs. 13.0 [11.0; 16.0] days (non-MAAE vs. MAAE, respectively) with no statistically significant difference between the groups. The median follow-up duration was 42.5 [15.0; 86.0] months $v s .70 .0$ [21.0; 88.0] months (nonMAAE $v s$. MAAE, respectively). The mean MAAE-free duration was $10.0[9.0 ; 19.0]$ months. The 8-year MAAEfree survival rate was $75.1 \%$ (Figure $3 A$ ). The 8 -year overall survival rate was $95.7 \%$.

\section{In-hospital mortality}

There were two cases of in-hospital mortality. Patients in both cases underwent partial arch replacement, and there was no primary intimal tear observed during surgery. One case of in-hospital mortality included a patient who died on postoperative day 15 due to aspiration pneumonia and 
Table 2 Univariable analysis for predictors of MAAE in preoperative computed tomography findings

\begin{tabular}{lccc}
\hline \multirow{2}{*}{ Variables } & \multicolumn{3}{c}{ Univariate analysis } \\
\cline { 2 - 4 } Male sex & HR & $95 \% \mathrm{Cl}$ & P value \\
Age & 1.844 & $0.492,6.903$ & 0.3637 \\
Smoking & 0.998 & $0.933,1.066$ & 0.9422 \\
HTN & 1.586 & $0.418,6.014$ & 0.4979 \\
AR & 0.321 & $0.085,1.207$ & 0.9270 \\
PAR & 0.718 & $0.176,2.930$ & 0.6444 \\
HT index_Max & 2.622 & $0.651,10.564$ & 0.1752 \\
${ }^{\dagger}$ HT index_IVC & 3.141 & $1.085,9.093$ & 0.0348 \\
HT index_CA & 1.920 & $0.877,4.203$ & 0.1029 \\
\hline
\end{tabular}

${ }^{\dagger}, \mathrm{HT}$ index_IVC = dHTR_IVC/aHTR. AR, ascending aorta replacement; aHT, hematoma thickness of ascending aorta; aHTR, hematoma thickness ratio of ascending aorta; aMax, maximal diameter of ascending aorta; $\mathrm{CA}$, celiac axis; $\mathrm{Cl}$, confidence interval; dHT, hematoma thickness of descending aorta; dHTR, hematoma thickness ratio of descending aorta; dMax, maximal diameter of descending aorta; HR, hazard ratio; HTN, hypertension; IVC, inferior vena cava; MAAE, major adverse aortic event; PAR, partial arch replacement.

sepsis. The other preoperatively demonstrated unstable vital signs and pulmonary edema. Following graft replacement, it was necessary to perform coronary artery intervention intraoperatively.

\section{Univariable analysis}

The results of the univariable analysis are summarized in Table 2. We performed simple Cox regression analysis, which revealed that HT index_Max was an independent predictor of MAAEs (HR, 3.141; 95\% CI: 1.085-9.093; $\mathrm{P}=0.0348)$. Other clinical variables and surgical variables were not significant predictors.

\section{Kaplan-Meier curve}

Using maximally-selected rank statistics analysis with $\mathrm{R}$ package to evaluate the best cut-off value, a Kaplan-Meier curve was plotted. The best cut-off values for HT index Max and HT index_IVC were 1.23 and 0.87 , respectively. Regarding freedom from MAAEs, a significantly higher MAAE rate was observed in patients with HT index Max $>1.23$ and HT index_IVC $>0.87$ (log-rank, $\mathrm{P}<0.001$ and $\mathrm{P}=0.014$, respectively) (Figure $3 B, C$ ). Patients with no intimal tears at the time of surgery had a worse prognosis (log-rank, $\mathrm{P}=0.014$ ) (Figure 3D).

\section{Comparison of aortic measurements according to the presence or absence of intimal tears found intraoperatively}

Table 3 shows the comparison of aortic measurements according to the presence or absence of intimal tears found during surgery. In the intimal tear positive $(+)$ group, the values measured in the ascending aorta are larger and the values measured in the descending aorta are smaller. Among them, dHT, dHTR, HT index_Max, dHT_IVC, and HT index_IVC showed significant differences between both groups. The incidence of MAAE was significantly lower in the intimal tear + group (intimal tear + group $v s$. intimal tear - group; $0 \%, \mathrm{n}=0$ vs. 29\%, $\mathrm{n}=9$, respectively).

\section{Profiles of MAAE and re-intervention}

The profiles of MAAEs and re-intervention are summarized in Figure 4. There were nine patients with MAAEs, of whom eight underwent re-intervention. There were seven patients who underwent thoracic endovascular aneurysm repair and one who underwent the false lumen procedure. False lumen procedure is defined as an endovascular procedure with closure of the communicating channels between the true lumen and false lumen or obliteration of the false lumen itself, of both the entry and re-entry tears, using various materials (arterial vascular plugs, coils, stents, 
Table 3 Comparison of aortic measurement in preoperative CT scan findings according to intimal tear in operative field

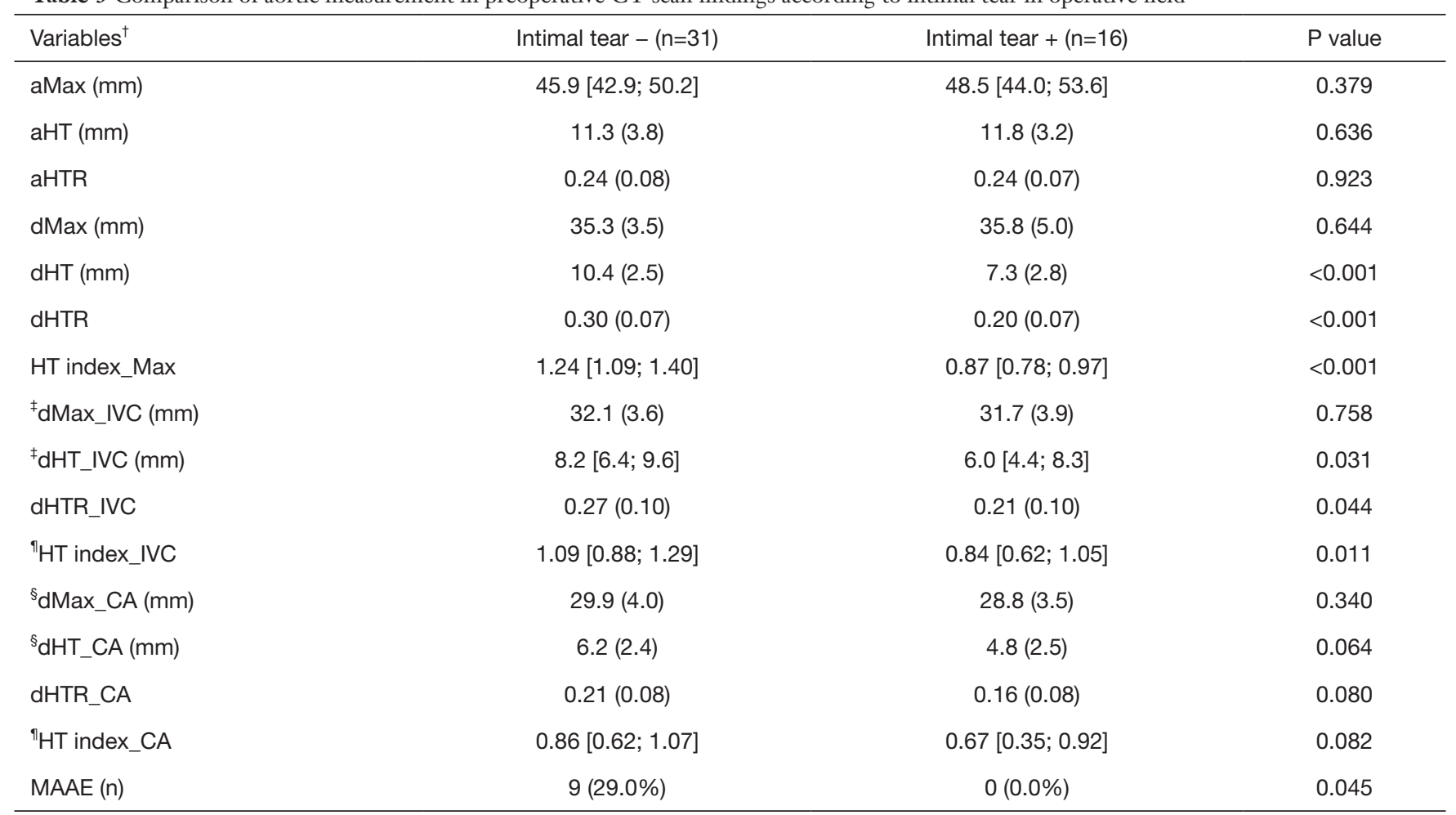

${ }^{\dagger}$, values expressed as means (standard deviations) for normally distributed continuous data, medians [IQRs: 25th percentile; 75th percentile] for non-normal distributed continuous data, or numbers (percentages) for categorical data; ${ }^{\ddagger}$, measurements were taken at the level of junction of inferior vena cava and right atrium; ${ }^{\S}$, measurements were taken at the level of celiac axis; " , HT index_IVC = dHTR_ IVC/aHTR; HT index_CA = dHTR_CA/aHTR. aHT, hematoma thickness of ascending aorta; aHTR, hematoma thickness ratio of ascending aorta; aMax, maximal diameter of ascending aorta; CA, celiac axis; CT, computed tomography; dHT, hematoma thickness of descending aorta; dHTR, hematoma thickness ratio of descending aorta; dMax, maximal diameter of descending aorta; IVC, inferior vena cava; MAAE, major adverse aortic event.
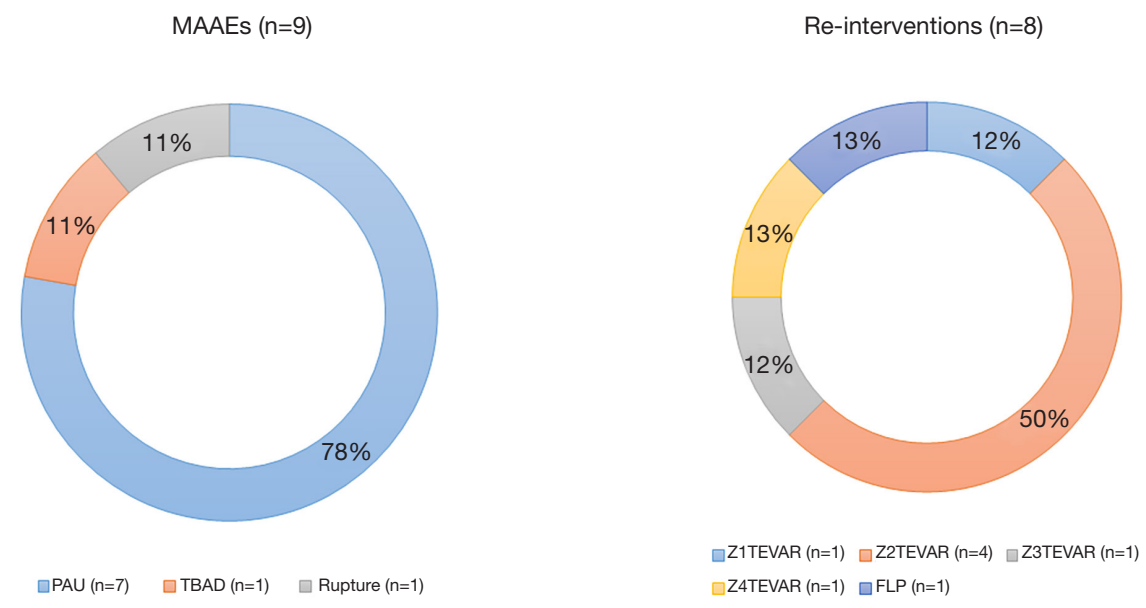

Figure 4 Profiles of MAAEs and re-intervention. FLP, false lumen procedure; MAAE, major adverse aortic event; PAU, penetrating aortic ulcer; TBAD, type B aortic dissection; TEVAR, thoracic endovascular aortic repair; Z, zone. 
and glues) (13). Fortunately, there were no patients who experienced MAAEs after the re-intervention and there was no post-procedural mortality. One patient with MAAEs did not receive re-intervention due to their refusal to undergo any procedure.

\section{Discussion}

Management of ATAIMH remains controversial. A systematic review of surgical outcomes of ATAIMH identified acceptable risks of postoperative mortality. However, significant heterogeneity exists in the outcomes between Western and Eastern countries (14). Western guidelines recognize IMH as a subset of acute aortic syndrome, and therefore advocate urgent surgery for ATAIMH. In contrast, some Asian guidelines consider IMH to be a distinct pathological process requiring a unique approach, such as medical treatment and delayed surgery (15-17). Indeed, both the 2010 ACC/AHA guidelines and 2014 European Society of Cardiology guidelines recommend urgent surgery $(7,18)$, whereas the 2011 Japanese Circulation Society recommends medical therapy for ATAIMH (19). Despite the different reported outcomes and recommendations from the Western and Eastern medical societies, the patients who demonstrate unstable hemodynamics, malperfusion syndrome, uncontrolled continuous pain, and progression to $\mathrm{AD}$ and rupture are candidates for emergency surgery within 1-2 days of symptom onset, ideally (20-23). Depending on the location of involvement, aortic repair is performed for restoring normal aortic dimension, where possible. A supracoronary anastomosis is performed in cases where the IMH does not involve aortic root (24). In general, hemiarch replacement is preferred, but total arch replacement is required if aortic arch aneurysm is present or if there is an intimal tear or PAU in the distal arch (16). In our study, surgical extent showed no differences between the two groups and was not predictors of MAAE. There exists no controversy regarding emergency surgery in patients with such an acute lifethreatening complicated form of ATAIMH, for which this study also demonstrated favorable short-term and long-term surgical outcomes. Matsushita $e t$ al. reported the surgical outcomes of ATAIMH. Five-year IMH-related eventfree rate was $89.1 \%$ and 5 -year $\mathrm{AD}$-related event-free rate was $85.1 \%$ (23). Roselli et al. reported the reintervention rate after surgical repair of $\mathrm{AD}$. In this study, 10-year reintervention rate was $38 \%$, with a cumulative incidence of $55 \%$ (25). Postoperative adverse events requiring reintervention included aortic rupture, pseudoaneurysm, rapidly growing aneurysmal change, graft infection, new dissection, and symptomatic chronic dissection $(23,25)$. Ten-year overall survival rate after AD surgery was $65 \%$. Reoperation during follow-up period was also related to significant morbidity and mortality. Risk factors for earlyand late-phase mortality include infected graft, concomitant coronary artery bypass grafting, higher body mass index, combined arch and descending/thoracoabdominal procedures, lower distal anastomosis site, and longer interval between reinterventions (25). Estrera et al. (26) reported early and late surgical outcomes of ATAIMH. In this study, after surgical repair of the proximal aorta, a typical intimal flap was noted in the distal aorta (descending or thoracoabdominal aorta) in 39\% (25 of 64) of patients. Another major source of controversy is the mechanism of generation of IMH. The aforementioned theory that describes IMH as a result of rupture of vasa vasorum rather than an intimal disruption and flow communication has not been scientifically validated. Moreover, it is questionable whether the pressure created by the rupture of vasa vasorum could overcome the pressure of the inner lumen (27). Some authors claim that all IMHs should be renamed as thrombosed type $\mathrm{AD}$ and that an intimal tear is always present but may not always be identified (5). Park et al. (6) reported prevalence of aortic intimal defect in surgically treated ATAIMH. At the time of surgery, small intimal tears were detected at the proximal aorta in 27 patients (73.0\%) although preoperative CT scan suggested no indication of intimal defect in 14 (51.9\%) of these patients. Another study (26) reported that intimal tear was noted intraoperatively in $66 \%$ (42 of 64) of patients with ATAIMH. In our study, although we planned to assess only pure ATAIMH without suggestive findings of intimal defect on preoperative CT, an intraoperative intimal tear was found in 16 out of 47 (34\%) patients. Total patients were divided into two groups according to the presence or absence of intimal tear, and the measurement of aorta was compared. In the intimal tear + group, the ascending aorta tended to be larger and the descending aorta to be smaller compared with the intimal tear - group, and dHT and dHTR were significantly smaller. Also, HT index_Max and HT index_ IVC were significantly smaller. The incidence of MAAE was significantly higher in the intimal tear + group. In the MAAE group of our study, the ascending aorta was smaller and the descending aorta was larger, compared with the non-MAAE group, and there were significant differences in HT index_Max and HT index_IVC. HT index_Max 
was an independent predictor of MAAE. Therefore, we believe that this aortic parameter can be utilized as evidence to infer the location of the primary intimal tear. In 2019, there was a two-patient case report of retrograde ATAIMH treated endovascularly (28). The first patient was hemodynamically stable, with prominent entry tear at the descending aorta, and the other had severe comorbidities along with the retrograde ATAIMH. Both patients underwent TEVAR and showed successful outcomes. In 2020, there was a multicenter study on TEVAR for retrograde ATAIMH. This is the first study to focus on the treatment of retrograde type A IMH with TEVAR. Eighteen non-consecutive selected patients with retrograde type A IMH with classical type B AD or type B IMH were treated with TEVAR. All procedures were technically successful. Median follow-up duration was 28.7 months and there was no overall mortality or 30-day mortality. Aortic remodeling was also favorable. Furthermore, the findings of this study confirm that TEVAR is a reasonable alternative treatment for eligible retrograde type A IMH patients (29). These findings indicate that the treatment strategy of retrograde ATAIMH could differ from antegrade ATAIMH, which means that it is important to know the location of the intimal tear. The limitations of this study must be considered when interpreting the results. This was a retrospective study, and the sample size was small. Additionally, all patients underwent emergency surgery; There was no control group. Therefore, we cannot suggest a novel algorithm of treatment modalities for ATAIMH. However, a prospective study is being planned.

\section{Conclusions}

In conclusion, HT index_Max was an independent predictor of MAAEs after ATAIMH surgery. Long-term periodical follow-up with early reintervention may, therefore, be necessary to improve outcome in these patients. As the optimal treatment method of ATAIMH is still controversial, inferring the location of the primary tear through the HT index can be helpful in determining the treatment method.

\section{Acknowledgments}

The authors thanks to http://www.editage.co.kr for diligent proofreading of this paper. This abstract was presented at the 34th EACTS annual meeting (Barcelona, Spain). Funding: None.

\section{Footnote}

Reporting Checklist: The authors have completed the STROBE reporting checklist. Available at https://dx.doi. org/10.21037/jtd-21-674

Data Sharing Statement: Available at https://dx.doi. org/10.21037/jtd-21-674

Peer Review File: Available at https://dx.doi.org/10.21037/ jtd-21-674

Conflicts of Interest: All authors have completed the ICMJE uniform disclosure form (available at https://dx.doi. org/10.21037/jtd-21-674). Suk-Won Song serves as an unpaid editorial board member of fournal of Thoracic Disease from Feb 2021 to Jan 2023. The other authors have no conflicts of interest to declare.

Ethical Statement: The authors are accountable for all aspects of the work in ensuring that questions related to the accuracy or integrity of any part of the work are appropriately investigated and resolved. The study was conducted in accordance with the Declaration of Helsinki (as revised in 2013). The study was approved by the Institutional Review Board of the Gangnam Severance Hospital, Yonsei University College of Medicine (Yonsei Institutional Review Board approval no. 3-2020-0317), and the requirement for obtaining individual consent was waived in this retrospective analysis.

Open Access Statement: This is an Open Access article distributed in accordance with the Creative Commons Attribution-NonCommercial-NoDerivs 4.0 International License (CC BY-NC-ND 4.0), which permits the noncommercial replication and distribution of the article with the strict proviso that no changes or edits are made and the original work is properly cited (including links to both the formal publication through the relevant DOI and the license). See: https://creativecommons.org/licenses/by-nc-nd/4.0/.

\section{References}

1. Krukenberg E. Beitrage zur Frage des Aneurysma dissecans. Beitr Pathol Anat Allg Pathol 1920;67:329-51.

2. Yu Y, Fei A, Wu Z, et al. Aortic intramural hemorrhage: A distinct disease entity with mystery. Intractable Rare Dis 
Res 2017;6:87-94.

3. Yanagaki S, Ueda T, Masuda A, et al. Detection of the intimal tear in aortic dissection and ulcer-like projection in intramural hematoma: usefulness of full-phase retrospective ECG-gated CT angiography. Jpn J Radiol 2020;38:1036-45.

4. Kitai T, Kaji S, Yamamuro A, et al. Detection of intimal defect by 64-row multidetector computed tomography in patients with acute aortic intramural hematoma. Circulation 2011;124:S174-8.

5. Uchida K, Imoto K, Karube N, et al. Intramural haematoma should be referred to as thrombosed-type aortic dissection. Eur J Cardiothorac Surg 2013;44:366-9; discussion 369.

6. Park KH, Lim C, Choi JH, et al. Prevalence of aortic intimal defect in surgically treated acute type A intramural hematoma. Ann Thorac Surg 2008;86:1494-500.

7. Hiratzka LF, Bakris GL, Beckman JA, et al. 2010 ACCF/AHA/AATS/ACR/ASA/SCA/SCAI/SIR/STS/ SVM Guidelines for the diagnosis and management of patients with thoracic aortic disease. A Report of the American College of Cardiology Foundation/American Heart Association Task Force on Practice Guidelines, American Association for Thoracic Surgery, American College of Radiology,American Stroke Association, Society of Cardiovascular Anesthesiologists, Society for Cardiovascular Angiography and Interventions, Society of Interventional Radiology, Society of Thoracic Surgeons, and Society for Vascular Medicine. J Am Coll Cardiol 2010;5 5:e27-e129.

8. Heo W, Song SW, Lee KH, et al. Surgery for acute Type I aortic dissection without resection of supra-aortic entry sites leads to unfavourable aortic remodelling. Eur J Cardiothorac Surg 2018;54:34-41.

9. Uchida T, Sadahiro M. Thoracic Endovascular Aortic Repair for Acute Aortic Dissection. Ann Vasc Dis 2018;11:464-72.

10. Goldberg JB, Kim JB, Sundt TM. Current understandings and approach to the management of aortic intramural hematomas. Semin Thorac Cardiovasc Surg 2014;26:123-31.

11. Grimm M, Loewe C, Gottardi R, et al. Novel insights into the mechanisms and treatment of intramural hematoma affecting the entire thoracic aorta. Ann Thorac Surg 2008;86:453-6.

12. Watanabe S, Hanyu M, Arai Y, et al. Initial medical treatment for acute type a intramural hematoma and aortic dissection. Ann Thorac Surg 2013;96:2142-6.

13. Kim TH, Song SW, Lee KH, et al. The effect of false lumen procedures during thoracic endovascular aortic repair in patients with chronic DeBakey type IIIB dissections. J Vasc Surg 2018;68:976-84.

14. Tian DH, Chakos A, Hirst L, et al. Surgery for type A intramural hematoma: a systematic review of clinical outcomes. Ann Cardiothorac Surg 2019;8:518-23.

15. Kitai T, Kaji S, Yamamuro A, et al. Clinical outcomes of medical therapy and timely operation in initially diagnosed type a aortic intramural hematoma: a 20-year experience. Circulation 2009;120:S292-8.

16. Sandhu HK, Tanaka A, Charlton-Ouw KM, et al. Outcomes and management of type A intramural hematoma. Ann Cardiothorac Surg 2016;5:317-27.

17. Rozado J, Martin M, Pascual I, et al. Comparing American, European and Asian practice guidelines for aortic diseases. J Thorac Dis 2017;9:S551-60.

18. Erbel R, Aboyans V, Boileau C, et al. 2014 ESC Guidelines on the diagnosis and treatment of aortic diseases: Document covering acute and chronic aortic diseases of the thoracic and abdominal aorta of the adult. The Task Force for the Diagnosis and Treatment of Aortic Diseases of the European Society of Cardiology (ESC). Eur Heart J 2014;35:2873-926.

19. JCS Joint Working Group. Guidelines for diagnosis and treatment of aortic aneurysm and aortic dissection (JCS 2011): digest version. Circ J 2013;77:789-828.

20. Schoenhoff FS, Zanchin C, Czerny M, et al. Aorta Related and All-cause Mortality in Patients with Aortic Intramural Haematoma. Eur J Vasc Endovasc Surg 2017;54:447-53.

21. Hata M, Hata H, Sezai A, et al. Optimal treatment strategy for type A acute aortic dissection with intramural hematoma. J Thorac Cardiovasc Surg 2014;147:307-11.

22. Svensson LG, Labib SB, Eisenhauer AC, et al. Intimal tear without hematoma: an important variant of aortic dissection that can elude current imaging techniques. Circulation 1999;99:1331-6.

23. Matsushita A, Fukui T, Tabata M, et al. Preoperative characteristics and surgical outcomes of acute intramural hematoma involving the ascending aorta: A propensity score-matched analysis. J Thorac Cardiovasc Surg 2016;151:351-8.

24. Erbel R, Aboyans V, Boileau C, et al. 2014 ESC Guidelines on the diagnosis and treatment of aortic diseases: Document covering acute and chronic aortic diseases of the thoracic and abdominal aorta of the adult. The Task Force for the Diagnosis and Treatment of Aortic Diseases of the European Society of Cardiology (ESC). Eur Heart J 2014;35:2873-926. 
25. Roselli EE, Loor G, He J, et al. Distal aortic interventions after repair of ascending dissection: the argument for a more aggressive approach. J Thorac Cardiovasc Surg 2015;149:S117-24.e3.

26. Estrera AL, Sandhu HK, Leake SS, et al. Early and late outcomes of acute type A aortic dissection with intramural hematoma. J Thorac Cardiovasc Surg 2015;149:137-42.

27. Lansman SL, Saunders PC, Malekan R, et al. Acute aortic syndrome. J Thorac Cardiovasc Surg 2010;140:S92-7;

Cite this article as: Kim MS, Kim TH, Lee H, Song SW, Heo W, Sim SA, Yoo KJ. Note the descending aorta: predictors of postoperative major adverse aortic event in pure acute type A intramural hematoma. J Thorac Dis 2021;13(8):4935-4946. doi: $10.21037 /$ jtd-21-674 discussion S142-S146.

28. Haenen FWN, Van Der Weijde E, Vos JA, et al. Retrograde Type A Intramural Hematoma Treated Endovascularly in Two Cases. Ann Vasc Surg 2019;59:312. e15-8.

29. Ryoi O, Lin CH, Chen JM, et al. Endovascular Repair for Retrograde Type A Intramural Haematoma with Intimal Tear in the Descending Thoracic Aorta. Eur J Vasc Endovasc Surg 2020;60:386-93. 